doi.org/10.22498/pages.26.2.85

\title{
An integrated proxy and simulation data initiative for the Holocene and the last deglaciation
}

\author{
Heather Andres' ${ }^{1}$ O. Bothe ${ }^{2}$, K. Rehfeld ${ }^{3}$, S. Wagner ${ }^{2}$, N. Weitzel ${ }^{4}$ and E. Zorita ${ }^{2}$ \\ Hamburg, Germany, 16-18 April 2018
}

Comparing climate proxy and simulation data is fraught with challenges: age and calibration uncertainties in climate proxies, missing or incomplete processes and uncertain boundary conditions for climate models, and differences between gridded and site data are just a few examples. For the climate of the Common Era, multiple initiatives have already addressed these issues (e.g. the PAGES 2k Network regional working groups). On transient timescales beyond the late Holocene, there have been only a few integrated activities. Comparisons on these longer time scales involve largescale changes in climate states without an equivalent during the Holocene. As such, they require methods that address both the amplitude and timing of background climate changes and account for additional processes. For example, comprehensive Earth System Models need to include changes in ice sheets and related ocean circulation changes during deglaciation. Likewise, proxy data for this period, such as lake or marine sediments, are generally less well replicated than their late Holocene counterparts (e.g. tree rings and historical documents), resulting in more uncertain climate signals (Laepple et al. 2017).

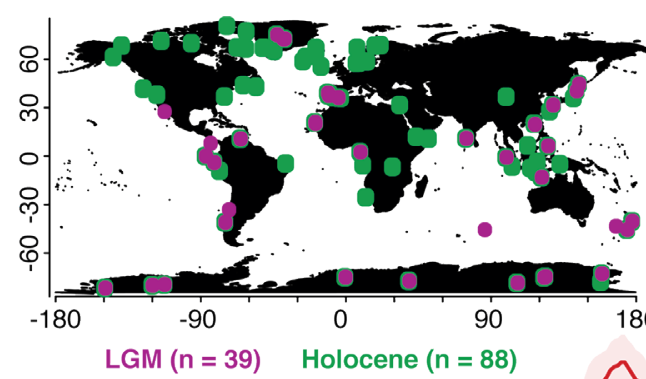

To address strategies for data-model comparisons on late Pleistocene and Holocene time scales, 30 participants, including global and regional climate modelers, statisticians and proxy experts, gathered in Hamburg for a three-day workshop. The meeting was cosponsored by the German climate modeling initiative PalMod. The workshop started with overview talks, which provided a solid base for breakout groups. These groups covered three main categories, addressing (i) conceptual aspects of data-model comparisons, (ii) inferring Holocene, and (iii) deglacial climate changes by combining proxies and models.

The methodological breakout group began to develop a framework based on the comparison of probability distributions of both proxy and simulated data, which accounts for quantifiable uncertainties. One of their main objectives was to develop summary metrics that assess the mismatch of reconstructed and simulated climate information and are robust with respect to uncertainties. The Holocene breakout group planned an integrated analysis of different types of proxy data and model simulations for not only hemispheric and global means, but

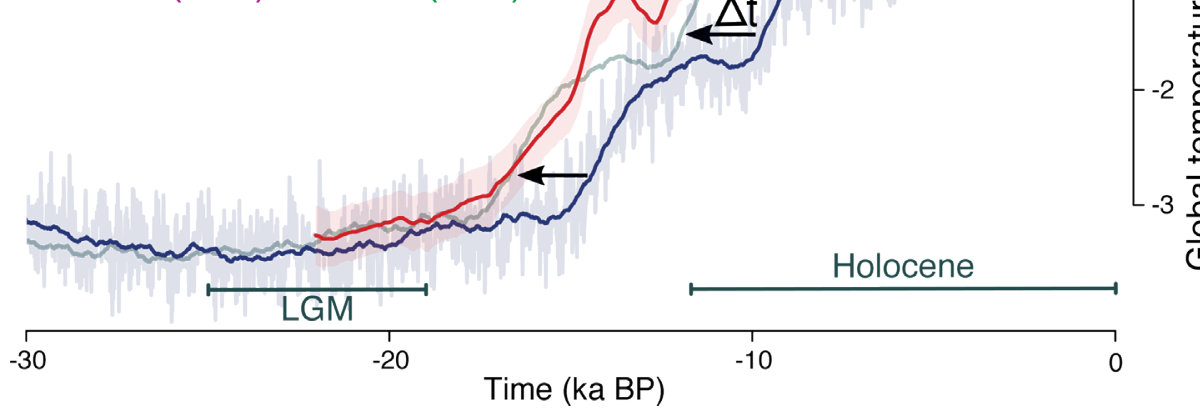

Figure 1: Comparison of an abrupt warming in global temperature during the deglaciation between climate model (blue line; Smith and Gregory 2012) and paleoclimate reconstruction data (red lines; Marcott et al. 2013 Shakun et al. 2012). The simulated time series is shifted in time to optimally match the reconstructed series for events of interest (grey line shows shifted series, horizontal arrows mark the time change $\Delta t$ ). The discrepancy, $\Delta T$, is evaluated at every available spatial location (see inset; dots show data availability during the Holocene, blue, and the LGM, red; Rehfeld et al. 2018). also regions, such as Europe and the North Atlantic. This group focused on apparent model-data mismatches beyond the so-called "Holocene Conundrum" (Liu et al. 2014), like the influence of large scale atmospheric and oceanic processes on regional Holocene climate variability, and discrepancies between the seasonality and regional characteristics of trends in simulations and pollen-based reconstructions. The deglaciation breakout group developed a procedure to evaluate the representation of largeamplitude events in proxies and simulations. This method compares the spatio-temporal structure of an event relative to its onset, and thus allows comparisons of manifestations of internal variability as well as events that occur at different times in the simulations and proxy records. The approach depends on metrics that quantify mismatches in time and space. This breakout group carried out initial testing of their methodology with existing datasets (Fig. 1) and will prepare, with the methodology group, a document with guidelines and a specific description of the algorithm (i.e. a cookbook) to enable others to implement the same procedure.

In addition to the methodological cookbook, workshop attendees planned future activities. These include writing an overview manuscript on the questions formulated in the Holocene breakout group. Additional follow-up plans include an interactive toolbox to compare model simulations with proxies using a hierarchy of metrics with varying complexity.

\section{AFFILIATIONS}

'Department of Physics and Physical Oceanography,

Memorial University of Newfoundland, St. John's,

Canada

Institute of Coastal Research, Helmholtz-Zentrum Geesthacht, Germany

${ }^{3}$ Institut für Umweltphysik, Universität Heidelberg, Germany

IInstitut für Geowissenschaften und Meteorologie, Universität Bonn, Germany

CONTACT

Oliver Bothe: oliver.bothe@hzg.de

REFERENCES

Laepple T et al. (2017) PAGES Mag 25: 140-141 Liu Z et al. (2014) PNAS 111: E3501-E3505 Marcott SA et al. (2013) Science 339: 1198-1201

Rehfeld K et al. (2018) Nature 554: 356-359

Shakun J et al. (2012) Nature 484: 49-54 Smith RS, Gregory J (2012) Clim Dyn 38: 1545-1559 